Apidologie, 1984, 15 (1), 33-42.

\title{
ÉTUDE COMPARÉE, EFFECTUÉE AU LABORATOIRE, DES APTITUDES AU BUTINAGE D'ABEILLES DE RACE APIS MELLIFICA LIGUSTICA ET D'HYBRIDES INTERRACIAUX APIS MELLIFICA (LIGUSTICA $\times$ CAUCASICA) $\times$ MELLIFICA
}

\author{
Minh-Hà PHAM-DELEGUE, Claudine MASSON et Ph. DOUAULT \\ Laboratoire de Neurobiologie Sensorielle de l'Insecte, I.N.R.A.-C.N.R.S. \\ Station de Recherches sur l'Abeille et les Insectes Sociaux \\ $F-91440$ Bures-sur-Yvette
}

\section{RESUME}

Afin de mieux comprendre certains des mécanismes à la base des différences existant dans l'activité de butineuses d'abeilles de race pure et d'abeilles hybrides, les auteurs ont entrepris au laboratoire une étude comparée du comportement de butinage au niveau d'une source alimentaire artificielle chimiquement connue (solution sucrée et odeur associée constantes), de l'hybride triple Apis mellifica (ligustica $\times$ caucasica) $\times$ mellifica et de l'abeille de race pure Apis mellifica ligustica (abeille italienne).

Il apparaît que le comportement de butinage de l'hybride triple se distingue de celui de labeille italienne par une phase de recrutement plus intense, rapidement consécutive à la découvertc de la source alimentaire par les première butineuses, et par un rythme plus rapide des visites. L'intensité de recrutement des hybrides, observée en conditions contrôlées, peut permettre d'expliquer leurs bonnes qualités sur le plan apicole et en tant qu'agents pollinisateurs.

\section{INTRODUCTION}

L'amélioration génétique de l'abeille, rendue possible par le développement des techniques d'insémination artificielle, a permis l'obtention d'abeilles présentant dans divers domaines des qualités supérieures à celles des abeilles communes : rendements en miel plus élevés, faible agressivité, bonne résistance aux maladies, faible tendance à l'essaimage, adaptation satisfaisante au climat et à la flore, etc. 
Des succès ont été obtenus par la méthode classique de sélection à l'intérieur d'une population notamment pour des qualités spécifiques, comme la résistance à la loque américaine (RothenbuhleR et THOMPSON, 1956), ou la récolte préférentielle de pollen de luzerne (MACKENSEN et NYE, 1966). Toutefois des qualités complexes, telles que l'aptitude à donner de fortes productions de miel, n'ont pu être améliorées de façon appréciable que grâce à l'utilisation de l'effet d'hétérosis résultant de certaines hybridations inter ou intra raciales. RutTNER (1968) a présenté les bénéfices obtenus dans la récolte de miel après différents croisements inter-raciaux et il apparaît que les résultats peuvent atteindre plus de $200 \%$ de ceux enregistrés pour la moyenne des différentes races ou le meilleur des parents. WaraKomsKa et Louveaux (1964) ont montré que des colonies d'hybrides interraciaux Apis mellifica $\times$ Apis mellifica ligustica ont une précocité et une activité supérieures par rapport à des abeilles de race noire pure Apis mellifica mellifica, ce qui se traduit par une récolte de pollen plus importante. Si l'on considère la valeur pollinisatrice des abeilles, Joubert et coll. (1977) ont établi que les abeilles hybrides Apis mellifica caucasica $\times$ ligustica sont 3 fois plus efficaces dans la pollinisation du trèfle violet que l'abeille noire Apis mellifica mellifica. GarY et coll., 1978 indiquent que des hybrides «Hy-Queen» ont des distances de butinage inférieures à celles d'abeilles italiennes, ce qui pourrait permettre de maintenir plus facilement des populations d'abeilles sur une aire à polliniser.

Une formule d'hybride à 3 voies, faisant intervenir 3 races d'abeilles différentes (ligustica $\times$ caucasica) $\times$ mellifica a permis d'obtenir de fortes récoltes de miel, une bonne homogénéité des colonies et une grande régularité de production au cours des années (Fresnaye et coll., 1974; Fresnaye et Lavie, 1976 ; Cornuet et Fresnaye, 1979).

Les qualités de l'abeille pour la production de miel ou la pollinisation des cultures sont liées à son activité de butinage. Cette aptitude au butinage peut être estimée par des mesures de l'activité de vol des abeilles, ainsi que l'ont fait KERR et coll. (1970) chez Apis mellifera ligustica, l'abeille africanisée et leur hybride $F_{1}$, montrant, chez cet hybride, la dominance des gènes africains sur les gènes italiens dans le contrôle du comportement de vol. Hohman (1970) a étudié chez des butineuses de pollen l'influence d'essences éthérées sur l'activité de recrutement estimée en fonction de la durée des danses. C'est en observant l'activité d'abeilles au niveau d'une source alimentaire artificielle, dont les caractéristiques varient (concentration en sucre, vitesse de distribution, température), que PFlumm (1969) estime la tendance des abeilles au butinage.

Dans ce travail, nous avons voulu comparer en conditions contrôlées l'activité de butinage d'abeilles de race pure et d'hybrides interraciaux en observant le recrutement de butineuses au niveau d'une source alimentaire odoriférante; le 
critère d'activité de recrutement est constitué par le nombre de visites par unité de temps. L'étude a porté sur les abeilles de race pure Apis mellifica ligustica et les hybrides triples Apis mellifica (ligustica $\times$ caucasica) $\times$ mellifica.

\section{MATÉRIEL ET MÉTHODES}

\section{Matériel}

Afin de reconstituer pour les abeilles des conditions expérimentales aussi proches que possible des conditions de vie à l'air libre, tout en contrôlant les facteurs de l'environnement, nous avons expérimenté dans une cage de vol en grillage de nylon (maille $2 \mathrm{~mm}$ ), occupant un volume de $12 \mathrm{~m}^{3}$ et placée dans une enceinte climatisée. Cette enceinte, inspirée du dispositif de van Praagh (1972), et décrite de façon détaillée par Douault (1978), est éclairée par une lumière dont le spectre s'étend de $300 \mathrm{~nm}$ à $700 \mathrm{~nm}$ (tube Philips TLM $40 \mathrm{w} / 33 \mathrm{RS}$ ); l'intensité lumineuse est fixée à 300 lux ce qui assure une activité de vol satisfaisante; la photopériode est de 12 heures de jour et 12 heures de nuit. La température de la salle est maintenue à $24{ }^{\circ} \mathrm{C} \pm 2^{\circ} \mathrm{C}$ et l'hygrométrie est de $55 \%$. Le renouvellement de l'air de l'enceinte est assuré par une tourelle d'extraction, à raison de 5 fois par heure.

Le comportement de récolte pouvant être favorisé par les interactions sociales [présence de la reine, du couvain... (FREE, 1977)], les expériences sont conduites à l'échelle de colonies entières, maintenues dans des ruchettes (dimensions extérieures $21 \times 21,5 \times 27 \mathrm{~cm}$ ) contenant 6 cadres. Les colonies comportent 3000 ouvrières de printemps (les expériences s'étant déroulées des mois de juillet à septembre) et d'une reine féconde âgée de 1 an; un seul cadre de provisions est mis à leur disposition, la présence de cadres vides pouvant favoriser la récolte de nourriture (RINDERER, 1982). Deux colonies d'abeilles italiennes Apis mellifica ligustica et deux colonies d'hybrides triples Apis mellifica (ligustica $\times$ caucasica) $\times$ mellifica ont ainsi été préparées de façon extemporanée et introduites successivement dans l'enceinte expérimentale. Chacune des colonies a séjourné en moyenne une semaine dans la cage de vol.

L'activité de butinage est observée au niveau de fleurs artificielles rassemblées dans un dispositif qui a été décrit par ailleurs (Pham-Delegue et Masson, 1982; Pham-Delegue, 1983).

Les signaux chimiques jouent un rôle particulièrement important dans l'orientation des abeilles vers une source alimentaire, Menzel (1967) et Kriston (1973), notamment, ayant démontré que les signaux olfactifs sont plus rapidement appris que les signaux visuels; de plus il a été montré que l'apport d'essences éthérées à une source d'eau sucrée entraîne le déclenchement de danses ou en augmente l'intensité (Lindauer, 1948; KasCHEF, 1957). C'est pourquoi les fleurs artificielles sont ici constituées d'une source odorante associée à une solution sucrée. L'odeur utilisée est celle du géraniol, substance qui entre dans la composition de certains arômes végétaux tels que l'arôme de la mélisse (Melissa officinalis) selon GUENTHER (1949) et LaWRENCE (1978) et qui constitue d'autre part l'élément le plus important pondéralement de la phéromone de Nassanov impliquée dans le marquage de sources alimentaires par l'abeille (Boch et SHEARER, 1962). La solution sucrée est une solution de saccharose à $50 \%$; en effet, dans des expériences de choix, ce sucre est préféré au glucose et au fructose (WaLler, 1972) et le préférendum de concentration établi par WaLler pour le saccharose se situe entre 30 et $50 \%$. Six fleurs artificielles sont disposées en périphérie d'un plateau de $50 \mathrm{~cm}$ de diamètre, en PVC gris, couleur neutre pour l'abeille (von FrISCH, 1959). Les abeilles ne peuvent différencier les fleurs artificielles à partir de repères visuels car celles-ci sont toutes identiques et entrânées par un lent mouvement de rotation du plateau expérimental ( $1 / 3$ tour/minute). La présence de plusieurs sources alimentaires permet à un plus grand nombre de butineuses de se nourrir simultanément et la rotation du dispositif assure leur répartition régulière sur les différents sites.

Le dispositif expérimental est placé dans la cage de vol à $80 \mathrm{~cm}$ du sol et à $1 \mathrm{~m} 50$ de la ruchette expérimentale. 


\section{Méthodes}

Pour chacune des 4 colonies expérimentales, nous avons opéré de la façon suivante : les abeilles sont familiarisées aux conditions de l'environnement expérimental (milieu confiné, espace de vol limité, éclairage artificiel, température constante, etc.) pendant 4 jours, au cours desquels elles sont entrainées à venir à l'emplacement du dispositif expérimental. Au cours de cette phase de pré-conditionnement, et en dehors des périodes d'expérience, les abeilles sont nourries ad libitum à l'aide d'un dispositif simplifié (flacon rempli de solution sucrée suintant par les orifices percés à sa base et coupelle de pollen broyé). Ce type de dispasitif est réservé au nourrissement des abeilles, qui par ailleurs ne reçoivent aucune nourriture dans la ruchette, et n'est pas adapté à l'observation du recrutement des butineuses.

L'expérience proprement dite se déroule le $5^{e}$ jour. Le dispositif expérimental présentant les fleurs artificielles est introduit à 12 heures GMT. A partir de la $1^{\mathrm{re}}$, le nombre des visites est noté par périodes d'observation de 5 minutes. Entre chaque période de notation, le plateau est retiré pendant 5 minutes. Les résultats portent sur 15 périodes d'observations successives. L'ensemble de l'expérience dure 3 heures 30 minutes environ.

\section{RESULTATS}

Le nombre des visites obtenu par période d'observation est reporté en fonction du temps; on peut ainsi tracer une courbe de recrutement pour chaque colonie. Les 4 courbes expérimentales sont présentées dans la figure 1 .

Il apparaît qu'en fonction du type d'abeilles considéré - race pure ou hybride - les tracés des courbes de recrutement diffèrent très nettement, tandis que pour un même type d'abeilles, les courbes sont analogues. Le phénomène de recrutement est donc caractéristique du type d'abeilles et présente le décours suivant :

- Pour les 2 colonies de race pure $\left(R p_{1}\right.$ et $\left.R p_{2}\right)$ l'augmentation du nombre des visites est très progressive et ce nombre se stabilise en moyenne à une dizaine de visites par période d'observation de 5 minutes.

- Pour les 2 colonies hybrides $\left(\mathrm{H}_{1}\right.$ et $\left.\mathrm{H}_{2}\right)$, au cours des premières périodes d'observation ( 5 périodes pour $\mathrm{H}_{1}$ et 8 périodes pour $\mathrm{H}_{2}$ ), les niveaux de visites se maintiennent à des valeurs analogues à celles obtenues pour les colonies de race pure (en moyenne une dizaine de visites). Puis on observe une augmentation intense du nombre des visites, qui passe de 10 visites/5 minutes à plus de 80 visites $/ 5$ minutes en 4 périodes (pour $\mathrm{H}_{1}$ ) à 6 périodes (pour $\mathrm{H}_{2}$ ) d'observation. Cette phase d'augmentation des visites se caractérise donc par une très forte pente qui est sensiblement du même ordre pour les colonies hybrides : 5,2 pour $\mathrm{H}_{1}$ et 3,4 pour $\mathrm{H}_{2}\left(^{*}\right)$. Puis le nombre de visites par période d'observation de

$\left({ }^{*}\right)$ Les pentes sont calculées sur la partie ascendante des courbes de recrutement; soit un segment $A B$ pris sur cette partie de courbe, de coordonnées $A\left(x_{1}, y_{1}\right)$ et $B\left(x_{2}, y_{2}\right)$, la valeur de la pente s'obtient par l'expression $\frac{y_{2}-y_{1}}{x_{2}-x_{1}}$. 
5 minutes se stabilise à une valeur élevée de 80 à 100 visites $/ 5$ minutes pour les 2 colonies.

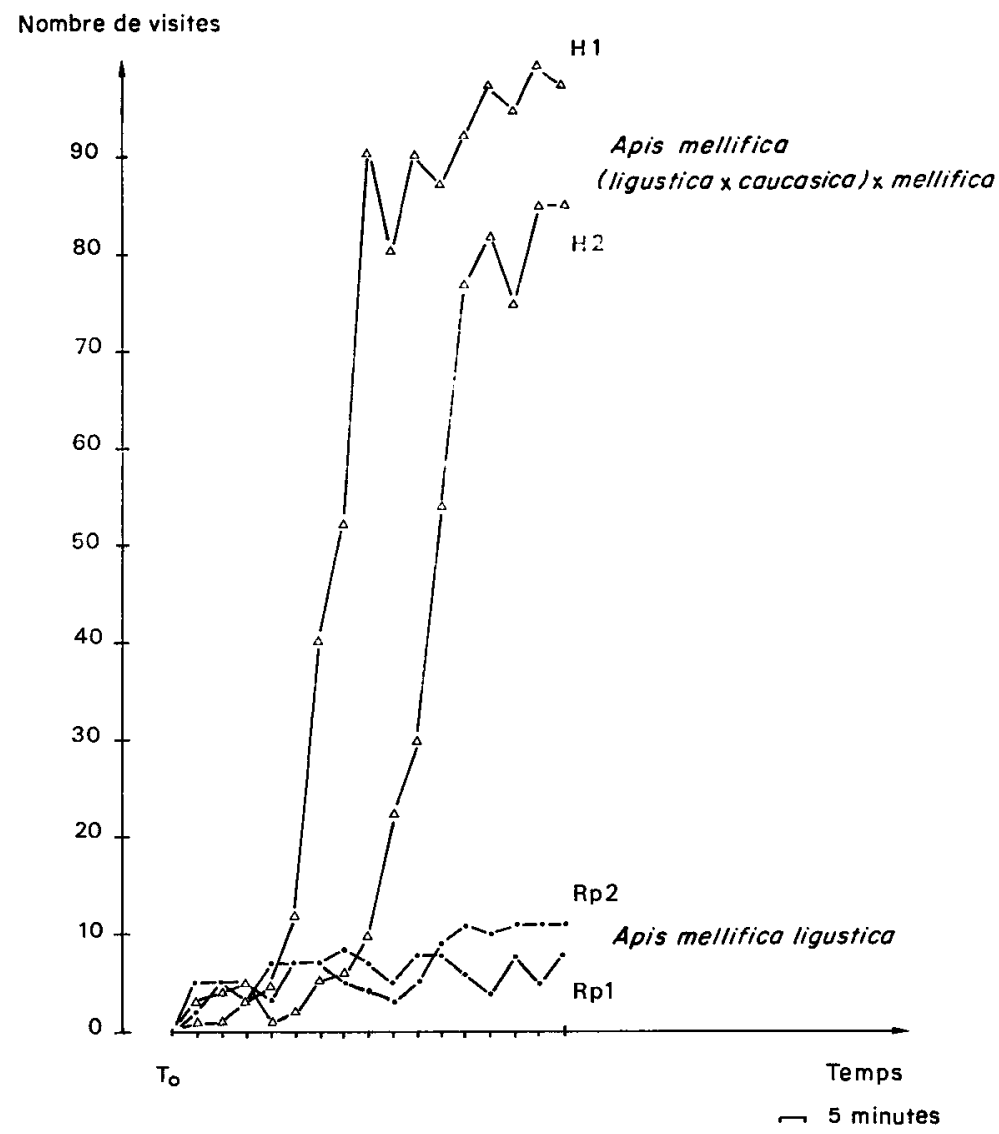

FIG. 1. - Recrutement de butineuses, évalué en nombre de visites au cours du temps sur une source alimentaire associée à du géraniol, pour deux colonies de race pure (Apis mellifica ligustica) et deux colonies hybrides [Apis mellifica (ligustica $\times$ caucasica) $\times$ mellifica]. $\mathrm{T}_{\mathrm{o}}=$ début des expérimentations.

$\mathrm{H}_{1}, \mathrm{H}_{2}=$ colonies hybrides.

$\mathrm{Rp}_{1}, \mathrm{Rp}_{2}=$ colonies de race pure.

(Les chiffres 1 et 2 se rapportent à l'ordre chronologique dans lequel les colonies ont été testées).

FIG. 1. - Recruitment of foragers estimated by the number of visits as a function of time, upon a food source associated with geraniol, for pure-bred colonies (Apis mellifica ligustica) and hybrid colonies [Apis mellifica (ligustica $\times$ caucasica) $\times$ mellifica].

$\mathbf{T}_{0}=$ beginning of experiments.

$\mathbf{H}_{1}, \mathrm{H}_{2}=$ hybrids colonies.

$\mathrm{Rp}_{1}, \mathrm{Rp}_{2}=$ pure-bred colonies.

(Numbers 1 and 2 are related to chronological order of testing). 
Il apparaît donc qu'à la fin de l'expérimentation (c'est-à-dire après 15 périodes d'observation, elles-mêmes espacées de 5 minutes), les abeilles hybrides recrutées se succèdent sur la source alimentaire à un rythme de plus de 80 visites $/ 5$ minutes alors que les abeilles de race pure ne dépassent pas un rythme de 10 visites $/ 5$ minutes.

\section{DISCUSSION}

Les résultats indiquent que, dans les conditions expérimentales décrites, le comportement de butinage de l'hybride triple se distingue de celui de l'abeille italienne par une phase de recrutement intense, rapidement consécutive à la découverte de la source alimentaire par les premières butineuses, et par un rythme élevé et stable de visites à cette source alimentaire. Un tel comportement de butinage s'avère très reproductible d'une colonie à l'autre, pour chaque type d'abeilles.

Les différences observées entre les 2 types d'abeilles dans les étapes de recrutement traduisent d'une part une meilleure adaptation des hybrides à des conditions d'élevage et de maintien en cage de vol, et à une situation artificielle de butinage.

D'autre part, le phénomène de recrutement observé chez l'hybride triple, ici en conditions contrôlées, caractérisé par une élévation rapide et une stabilisation à un nombre élevé de visites à une source alimentaire, permet de mieux comprendre les bonnes performances de cette abeille en plein champ : en effet, un type d'abeille dont les butineuses se succèdent plus rapidement sur les fleurs aura une plus grande efficacité en tant qu'agent pollinisateur et pourra rapporter des quantités supérieures de nectar à la ruche, donc produire davantage de miel ainsi que le montrent FRESNAYE et coll., 1974. L'hybridation apporte ici des résultats satisfaisants; il faut toutefois souligner que l'aptitude à la combinaison entre différentes races est très variable, et toutes les hybridations n'entraînent pas forcément d'effet d'hétérosis (RutTner cité par Louveaux, 1967 ; CoRnUET et Fresnaye, 1979).

Dans cette étude comparée du comportement de butinage de 2 types d'abeilles, le critère de comparaison choisi est le nombre de visites par unité de temps, indépendamment des individus qui effectuent ces visites. Or des études du phénomène de recrutement, menées dans les mêmes conditions expérimentales (Pham-Delegue, 1983) montrent que les individus recrutés en cage de vol par une source alimentaire définie (dont les caractéristiques de position, odeur, couleur, forme, ne varient pas) sont les mêmes au cours de plusieurs vols de butinage successifs ; des travaux anciens ont établi de tels résultats en conditions naturelles (von Frisch, 1923, 1965 ; RibBands, 1954). 
Par ailleurs, les aptitudes au butinage, définies ici selon l'intensité du recrutement, font également intervenir les préférences alimentaires et les capacités d'apprentissage des butineuses ; or celles-ci peuvent varier sensiblement en fonction de la race considérée.

Ainsi des différences raciales ont été mises en évidence dans les réponses à des signaux olfactifs ou visuels, avec ou sans conditionnement (KolTERManN, 1973 ; MENZEL et coll., 1973). On peut donc envisager de sélectionner les abeilles non seulement sur leurs aptitudes à visiter spécifiquement certaines cultures (voir MACKENSEN et NYE, 1966) mais aussi sur leurs capacités d'apprentissage. OTt et BrucKNer (1980) démontrent que des abeilles non consanguines apprennent mieux que des abeilles consanguines. Il serait donc intéressant de connaître les performances d'apprentissage d'abeilles hybrides et de savoir dans quelle mesure ces capacités sont liées à leur niveau d'activité, et c'est dans ce sens que nous envisageons de poursuivre ultérieurement le présent travail.

Reçu pour publication en octobre 1983. Accepté pour publication en janvier 1984.

\author{
SUMMARY \\ COMPARATIVE LABORATORY STUDY OF FORAGING ABILITIES OF HONEYBEES \\ APIS MELLIFICA LIGUSTICA AND INTERRACIAL HYBRIDS APIS MELLIFICA \\ $(L I G U S T I C A \times C A U C A S I C A) \times M E L L I F I C A$
}

Honeybee value, both in the field of bee-keeping (honey yield) and in the field of crop pollination, depends upon its foraging behavior. Genetic attempts for bee improvement have led to the creation of highly productive interracial hybrids.

In order to understand some mechanisms which determine better qualities of hybrids, foraging behavior of the three lines hybrid Apis mellifica (ligustica $\times$ caucasica) $\times$ mellifica and of the pure-bred bee Apis mellifica ligustica, have been compared under laboratory conditions.

Experiments have been carried out in a flight room on whole colonies of about 3000 workers. Two colonies of each type of bee have been tested successively. In order to take into account chemical signals, which are of the first importance in foraging behavior of honeybees, bee-plant relationships have been simulated using a device which comprised artificial flowers (a sugary solution associated with a scent), and which allowed elimination of visual signals.

The record of number of forager visits as a function of time has allowed establishment of recruiting curves. It appears (fig. 1) that the recruiting curves are specific for the type of bee considered :

- for the pure-bred honeybee, the number of visits increases gradually and reaches a steady state of 10 visits per observation period of 5 minutes;

- for the hybrid bee, recruiting is characterized by a rapid increase in the number of visits, followed by a steady state of 80 visits per observation period of 5 minutes.

The intense recruiting phenomenon seen among hybrid foragers not only indicates their ability to adapt to artificial conditions, but allows a better understanding of the superior performance 
of these bees in the field. Rapid recruitment leads to greater pollination and nectar-gathering efficiency. This in turn provides larger crop and honey yields. Further studies are needed to determine how behavior within the hive influences such rapid recruitment of foragers.

\section{ZUSAMMENFASSUNG}

\section{VERGLEICHENDE LABORUNTERSUCHUNGEN DER SAMMELLEISTUNGEN VON BIENEN DER RASSE A.M. LIGUSTICA UND VON RASSENHYBRIDEN A.M. (LIGUSTICA $\times$ CAUCASICA) $\times$ MELLIFICA}

Die Leistungen der Bienen in der Imkerei (Honigertrag) und bei ihrem Einsatz als Bestäuber von Blüten hängen beide von ihrem Sammelverhalten $a b$. Versuche zu einer genetischen Verbesserung der Bienen mit dem Ziel eines gesteigerten Honigertrages haben zu sehr leistungsfähigen Rassenhybriden geführt.

Um einige der Mechanismen besser zu verstehen, welche den höheren Leistungen der Hybriden zugrunde liegen, wurde das Sammelverhalten der dreifachen Hybride Apis mellifica (ligustica $\times$ catcasica) $\times$ mellifica und der reinrassigen Apis mellifica ligustica unter Laborbedingungen miteinander verglichen.

Die Experimente wurden in einem Flugraum mit kompletten Völkchen mit 3000 Bienen durchgeführt. Zwei Völker von jedem Bienentyp wurden nacheinander getestet. Um chemische Signale einzubeziehen, die für das Sammelverhalten der Biene in erster Linie von Bedeutung sind, und um eine Bienen-Blütenbeziehung zu simulieren, wurden in der Versuchsanordnung «künstliche Blüten» (Zuckerlösung zusammen mit einem Duft) verwendet und gleichzeitig visuelle Signale vermieden. Die Registrierung der Zahl der Besuche der künstlichen Futterstelle in einem bestimmten Zeitabschnitt ermöglichte die Aufstellung von Rekrutierungskurven.

Es scheint, daß die Rekrutierungskurven für jeden untersuchten Bienentyp spezifisch sind (fig. 1) :

- bei der reinrassigen Ligustica steigt die Zahl der Besuche steil an und stabilisiert sich dann bei einer Zahl von 10 Besuchen während der Beobachtungszeit von 5 Minuten;

- bei der Hybridbiene wird die Rekrutierung durch einen steilen und hohen Anstieg der Zahl der Besuche charakterisiert, gefolgt von einer steten Phase mit 80 Besuchen je Beobachtungszeit von 5 Minuten.

Eine derart intensive Rekrutierung der Hybridbienen bringt zum einen ihre bessere Anpassungsfähigkeit an die künstlichen Versuchsbedingungen zum Ausdruck, zum anderen führt es zu einem besseren Verständnis der guten Leistungen dieser Bienen im Feld : Bienen, die einander rascher und in größerer Zahl zu den Blüten folgen, sollten eine höhere Leistung als Bestäuber und größere Honigernten bringen. Weitere Untersuchungen sollen über die diesem Sammelverhalten zugrunde liegende Lernfähigkeit Aufschluß geben.

\section{BIBLIOGRAPHIE}

Boch R., Shearer D.A., 1962. - Identification of geraniol as the active component in Nassanoff pheromone of the honey bee. Nature, 194, 704-706.

Cornuet J.M., Fresnaye J., 1979. - Production de miel chez des hybrides interraciaux d'abeilles (Apis mellifica L.) lors de générations successives de rétrocroisements sur la race locale. Apidologie, 10 (1), 3-15. 
Douault Ph., 1978. - Lélevage expérimental des abeilles en milieu clos. La nouvelle salle d'élevage de la Station de Recherches de Bures-sur-Yvette. Bull, tech. Apic., OPIDA, 5 (4), 15-22.

FrEE J.B., 1977. - The social organization of honeybees. Edward Arnold, London, 67 p.

Fresnaye J., Lavie P., Boesiger E., 1974. - La variabilité de la production de miel chez l'Abeille de race noire (Apis mellifica mellifica) et chez quelques hybrides interraciaux. Apidologie, 5 (1), 1-20.

Fresnaye J., Lavie P., 1976. - Sélection et hybridation de labeille en France. Bull. tech. Apic., OPIDA, 3 (4), 15-20.

Frisch K. von, 1923. - Über die «Sprache» der Bienen, eine tierpsychologische Untersuchung. Zool. Jahrb. (Physiol.), 40, 1-186.

Frisch K. von, 1959. - Aus dem Leben der Bienen. Springer Verlag, Berlin, 179 p.

FriscH K. von, 1965. - Tanzsprache und Orientierthng der Bienen. Springer Verlag, Berlin, Heidelberg, New York, 578 p.

Gary N.E., Witherell P.C., Lorenze N.K., 1978. - The distribution and foraging activities of common Italian and «Hy-Queen» honeybees during Alfalfa pollination. Environ. Entomol., 7 (2), 233-240.

Guenther E., 1949. - The essential oils. 3 - Individual essential oils of the plant families Rutaecae and Labiatae. New York: D. Van Nostrand Co., Inc.

Hohmann H., 1970. - Uber die Wirkung von Pollen-extrakten und Duftstoffen auf das Sammelund Werbeverhalten höselnder Bienen (Apis mellifica L.). Apidologie, 1 (2), 157-178.

Joubert G., Tasei J.N., Delaude A., 1977. - Pollinisation du trèfle violet diploïde (Trifolium pratense L.) par les Apoïdes et particulièrement par les abeilles domestiques hybrides (Apis mellifica caucasica $\times$ Apis mellifica ligustica). Apidologie, 8 (3), 259-280.

Kaschef A.H., 1957. - Über die Einwirkung von Duftstoffen auf die Bienentänze. Z. 1gl. Physiol., 39, $562-576$.

Kerr W.E., Goncalvez L.S., Blotta L.F., Maciel H.B., 1970. - Biologia comparada de abelhas italianas (Apis mellifera ligustica), abelhas af:icanizadas (Apis mellifera adansonii) e suas hibridas. Anais do le Congr. Bras. Apic., 151-185.

Koltermann R., 1973. - Rassen- bzw. artspezifische Duftbewertung bei der Honigbiene und ökologische Adaptation. J. comp. Physiol., 85, 327-360.

KRISTON 1., 1973. - Die Bewertung von Duft- und Fa:bensignalen als Orientierungshilfen an der Futterquelle durch Apis mellifera L. J. comp. Physiol., 84, 77-94.

Lawrence B.M., 1978. - Progress in essential oils, Perfurmer Flavorist, 16 (3), 54-58.

Lindauer M., 1948. - Über die Einwirkung von Duft- und Geschmacksstoffen sowie anderer Faktoren auf die Tänze der Bienen, Z. Igl. Physiol., 31, 348-412.

Louvenux J., 1967. - Les problèmes posés par la génétique et la sélection de l'abeille. Ann. Abeille, 10 (4), 213-252.

Mackensen O., NyE W.P., 1966. - Selecting and breeding for collecting alfalla pollen. J. apic. Res., 5, 79-86.

Menzel R., 1967. - Untersuchungen zum Erlernen von Spektralfarben durch die Honigbiene (Apis mellifica). Z. vgl. Physiol., 56, 22-62.

Menzel R., Freudel H., Rüht U., 1973. - Rassenspezifische Unterschiede im Lernverhalten der Honigbiene (Apis mellifica L.). Apidologie, 4 (1), 1-24.

OTT I., Brückner D., 1980. - Genetisch bedingte Unterschiede im Lernverhalten der Honigbiene (Apis mellifica carnica). Apidologie, 11 (1), 3-15.

Pfiumm W., 1969. - Beziehungen zwischen Putzverhalten und Sammelbereitschaft bei der Honigbiene. Z. vgl. Physiol., 64, 1-36.

Pham-Delegue M.H., 1983. - Etude par conditionnement associatif des paramètres olfactifs qui déterminent le comportement alimentaire sélectif chez l'abeille (Apis mellifica L.). Thèse 3. cycle, Neurosciences, Université Pierre et Marie Curie, Paris 6, 152 p. 
Pham-Delegue M.H., Masson C., 1984. - Etude par conditionnement associatif de l'attraction des abeilles par les arômes végétaux. Bull. Soc. Entomol. Fr., 10 p. (sous presse).

Ribbands C.R., 1954. - Communication between honeybees. I. The response of crop-attached bees to the scent of their crop. Proc. r. Entomol. Soc. London (A), 29, 10-12.

Rinderer T.E., 1982. - Regulated nectar harvesting by the honeybee. J. apic. Res., 21 (2), 74-87.

Rothenbuhler W.C., Thompson V.C., 1956. - Resistance to American Foulbrood in honeybees. I. Differential survival of larvae of different genetic lines. J. econ. Entomol., 49, 470-475.

RUTTNER F., 1968. - Methods of breedings honeybees : intra-racial selection or inter-racial hybrids ? Bee World, 49 (2), 66-72.

Van Praagh J.P., 1972, - Towards a controlled environment room suitable for normal colony life of Honeybees. J. apic. Res., II, 77-87.

WALLER G.D., 1972. - Evaluating responses of honeybees to sugar solutions using an artificialflower feeder. Ann. entomol. Soc. Am., 65 (4), 857-861.

Warakomska Z., Louveaux J., 1964. - Recherche sur les variatons du comportement de butinage chez des colonies d'abeilles de races différcntes. Ann. Abeille, 7 (3), 217-231. 